\title{
Commissioning of the Inner Tracker of the KLOE-2 experiment
}

\author{
A. Di Cicco ${ }^{\dagger}$, G. Morello ${ }^{\ddagger}$
}

On behalf of the KLOE-2 Collaboration

The KLOE-2 experiment is undergoing commissioning at the DA $\Phi$ NE $e^{+} e^{-}$collider of the Frascati National Laboratory of the INFN, after the integration of new detectors in the former KLOE apparatus. The Inner Tracker, a very light detector (material budget $<2 \% X_{0}$ ), is one of the new subdetectors and it is composed of 4 cylindrical triple-GEM with a stereo $\mathrm{X}-\mathrm{V}$ strips/pads readout. The commissioning phase of the Inner Tracker consists in the characterization of the detector response and in its performance evaluation. The method used to evaluate its detection efficiency is reported, together with some preliminary results.

PACS numbers: 29.40.Cs, 29.40.Gx

\section{Introduction}

After the integration of the new subdetectors [1, 2, 3, 4, 5] in the KLOE apparatus [6], the commissioning phase of the KLOE-2 Inner Tracker (IT) has started. This detector is composed of 4 cylindrical triple-GEM coaxial layers [7], equipped each with a double-view XV-strips/pads readout circuit. The dimension of the active area $\left(\sim 700 \times 300 \mathrm{~mm}^{2}\right)$ pushed the GEM deliverer (CERN TE-MPE-EM workshop), in collaboration with INFN-LNF groups, to proceed with a new manufacturing technique [8], while the assembly procedure has been totally developed at the Frascati National Laboratory (LNF) of the INFN. The Inner Tracker has been installed between the beam pipe and the inner wall of the KLOE Drift Chamber (DC) and will increase the acceptance for low transverse momentum tracks and improve charged vertex reconstruction (especially useful for events with more than two tracks originating from Interaction Point [9]). The whole KLOE-2 [10] apparatus works in a $0.52 \mathrm{~T}$ magnetic field. Although each layer has been tested in a dedicated area at LNF, before their integration in the IT,

\footnotetext{
${ }^{\dagger}$ Roma Tre University, Rome

‡ LNF - INFN, Frascati
} 
new tests with the detector in its final position are required. The IT commissioning benefits from the presence of the DC and its excellent tracking performance.

\section{Track reconstruction with the Inner Tracker}

Starting from DC hits, tracks are reconstructed including IT hits by using the Kalman filter. Inner Tracker must be aligned and calibrated in order to get the best tracking performance. The presence of the B-field influences the reconstruction since the signal electron cloud experiences the Lorentz force and therefore there is a shift in the position of the fired readout strips with respect to the true position of the intersection between the particle track and the readout plane. Spatial resolution is also affected by nonradial tracks crossing the detector as shown in fig. 2 27 . The combination of the two effects produces a focusing or a defocusing of the electron cloud according to the impact parameter of the track on the cylindrical detectors. These effects must be studied independently: cosmic-ray muon data will be used to evaluate the non-radial correction (without B-field) and the magnetic field influence (with B-field), and Bhabha scattering events will be used to check the effect of the two corrections. The calibration and alignment procedure are presently ongoing. In the next paragraphs the monitoring of the detector status and the efficiency evaluation will be described.

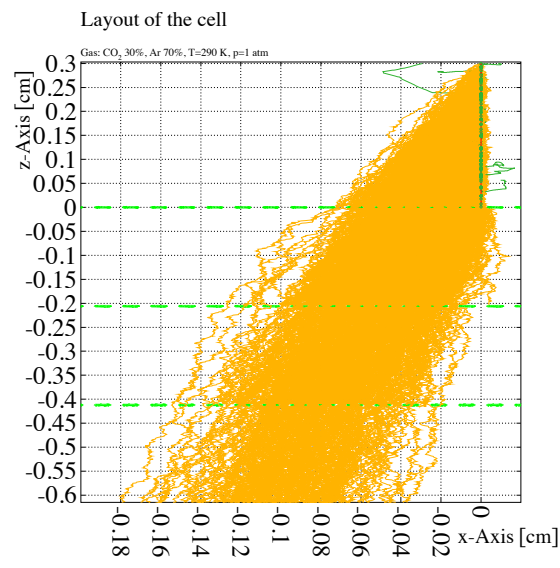

Fig. 1. GARFIELD simulation of electrons drift in a $0.52 \mathrm{~T}$ magnetic field with $\mathrm{Ar}: \mathrm{CO}_{2} 70$ : 30 gas mixture: the track is at the position $x=0$.
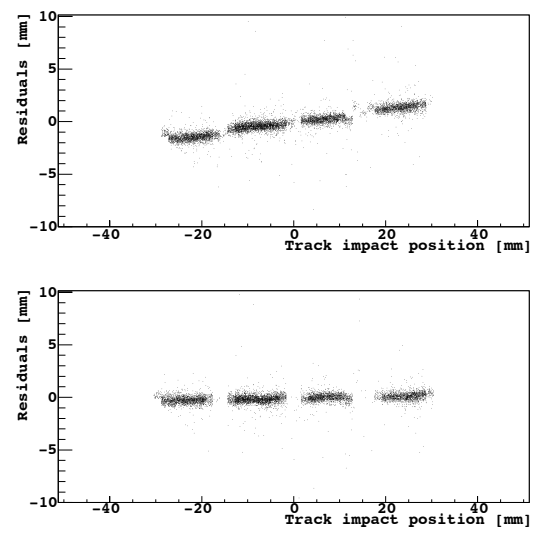

Fig. 2. Non-radial tracks effect on the residuals measured with a cylindrical GEM prototype. 


\section{Noisy and inefficient strips}

The detector status must be monitored in time and taken into account at the analysis stage. The definition of noisy or dead strips is related to their average occupancy for a single view and for each layer of the Inner Tracker. The technique used to evaluate the average occupancy and the definition of the strips status is briefly reported.

$\boldsymbol{X}$-view. The $\mathrm{X}$-view is given by the axial strips which provide coordinates in the $r-\varphi$ plane (transverse plane with respect to the beam line). All these strips have the same length and width (and consequently the same parasitic capacitance) so that, assuming a uniform exposition to cosmicray muons, the profile of the occupancy distribution is expected to be a gaussian function. In fig. 3 the occupancy for the $\mathrm{X}$-view strips is shown while its profile distribution is displayed in fig. 4. This latter is fitted with a combination of a second-degree polynomial (whose parameters are $p_{0}, p_{1}$ and $p_{2}$ ) and a gaussian function. The mean value, $\bar{\xi}$, of the gaussian fit

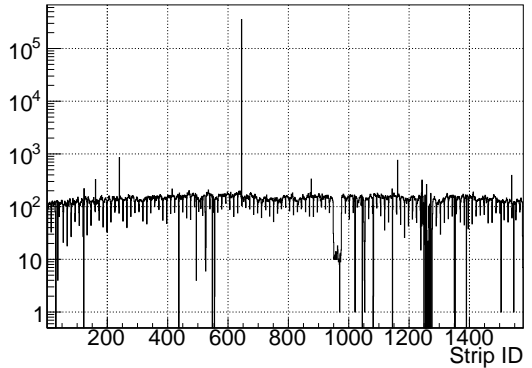

Fig. 3. Layer 2 X-view occupancy distribution.

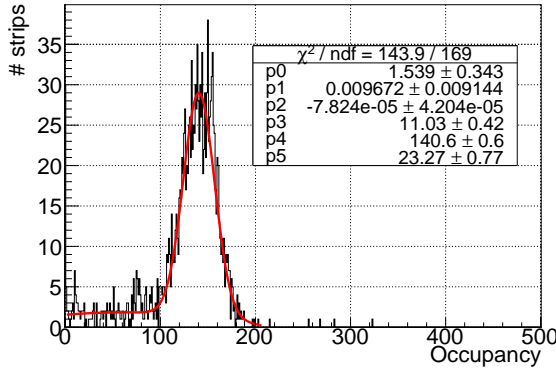

Fig. 4. Layer 2 X-view occupancy profile.

is considered to be the average occupancy of the strips belonging to the layer under study, while the sigma, $\sigma$, is used to identify the strips status as follows: let $\xi_{i}$ be the occupancy of the $i$-th strip, then strips with $\xi_{i}=0$ will be considered dead, while strips with $\xi_{i}>\bar{\xi}+5 \cdot \sigma$ will be considered noisy. The same definitions are applied to $\mathrm{V}$-view strips, although an intermediate step is required as further explained.

$\boldsymbol{V}$-view. V-view strips have different lengths and, thus, a non-uniform occupancy distribution. In order to use the tool previously described it seems natural to normalize the occupancy of the $i$-th strip to its length and then use the same definitions as for the axial strips.

The list of noisy strips is then recored in the database in order to mask them directly at the DAQ initialization stage for data taking and to monitor the time evolution of the noisy and dead strips. From a reference cosmic-ray run we have $\sim 6 \%$ of noisy strips and $\sim 17 \%$ of dead strips considering both 


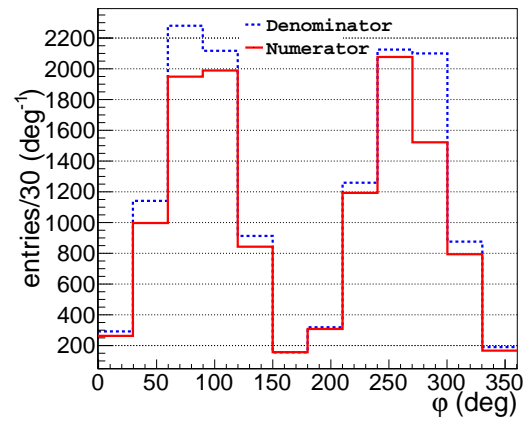

Fig. 5. Layer 2 distribution of $\varphi$ position of expected clusters (dashed blue line) and measured clusters (solid red line).

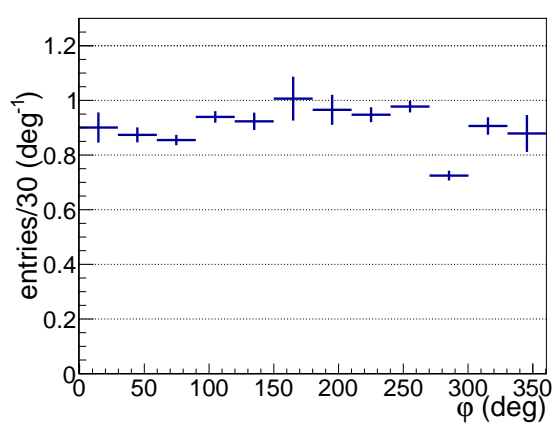

Fig. 6. Layer 2 efficiency as a function of the $\varphi$-position in the bending plane.

views and all the four layers.

\section{Inner Tracker Efficiency}

The Inner Tracker efficiency is evaluated by using cosmic-ray muon tracks reconstructed in the Drift Chamber in $0.52 \mathrm{~T}$ magnetic field.

Normalization sample. Tracks reconstructed in the DC are extrapolated to the Inner Tracker (IT) assuming straight lines, approximation valid if tracks with transverse momentum $p_{T}>500 \mathrm{MeV}$ are selected. Two crossing points with each IT layer and a Point of Closest Approach (PCA) of the track to the beam-line are required, with $z_{P C A}<35 \mathrm{~cm}$ and $R_{P C A}<5 \mathrm{~cm}$, the latter being the PCA radius in the bending plane. The two crossing points represents the track expected positions on the IT and their angular $\varphi$-position distribution in the bending plane will be the normalization sample.

Efficiency evaluation. The efficiency is evaluated as the ratio between the expected and the measured $\varphi$-position distributions. The latter distributions are obtained by using an algorithm which produces the 3-dimensional coordinates of the detected clusters. With the position of the reconstructed clusters we can compute the $\varphi$-position in the bending plane. The numerator of the efficiency is, therefore, the distribution of the measured clusters in the IT (fig. 5). A normalization sample of about $6 \times 10^{6}$ events in which at least a reconstructed track is present has been used to evaluate efficiency. Selection criteria reduced the normalization sample to $\sim 3.4 \times 10^{3}$ events, while $\sim 3.3 \times 10^{3}$ events enter the numerator. Layer 2 efficiency as a function of the $\varphi$-position is shown in fig. 6. Efficiency values are different from bin to bin, depending on the percentage of dead and noisy strips. 


\section{Conclusions}

The commissioning of the Inner Tracker for the KLOE-2 experiment is in progress. The detector operational condition is under optimization and tools to monitor the detector status and measure its efficiency have been developed. Preliminary efficiency measurements have been reported. Calibration and alignment procedures are under development and will use both cosmic-ray muon and Bhabha scattering events.

\section{Acknowledgements}

We warmly thank our former KLOE colleagues for the access to the data collected during the KLOE data taking campaign. We thank the DA $\Phi N E$ team for their efforts in maintaining low background running conditions and their collaboration during all data taking. We want to thank our technical staff: G.F. Fortugno and F. Sborzacchi for their dedication in ensuring efficient operation of the KLOE computing facilities; M. Anelli for his continuous attention to the gas system and detector safety; A. Balla, M. Gatta, G. Corradi and G. Papalino for electronics maintenance; M. Santoni, G. Paoluzzi and R. Rosellini for general detector support; C. Piscitelli for his help during major maintenance periods. This work was supported in part by the EU Integrated Infrastructure Initiative Hadron Physics Project under contract number RII3-CT- 2004-506078; by the European Commission under the 7th Framework Programme through the 'Research Infrastructures' action of the 'Capacities' Programme, Call: FP7-INFRASTRUCTURES-2008-1, Grant Agreement No. 227431; by the Polish National Science Centre through the Grants No. DEC2011/03/N/ST2/02641, 2011/01/D/ST2/00748, 2011/03/N/ST2/02652, 2013/08/M/ST2/00323, and by the Foundation for Polish Science through the MPD programme and the project HOMING PLUS BIS/2011-4/3.

\section{REFERENCES}

[1] D. Babusci et al., The low energy tagger for the KLOE-2 experiment, Nucl. Instr. and Meth. A 617 (2010) 81.

[2] F. Archilli et al., Gamma-gamma tagging system for KLOE-2 experiment, Nucl. Instr. and Meth. A 617 (2010) 266.

[3] A. Balla et al., The cylindrical GEM detector for the KLOE-2 Inner Tracker, JINST 9 C01014 (2014).

[4] F. Happacher et al., Tile and crystal calorimeter for the KLOE-2 experiment, Nucl. Phys. Proc. Suppl. 197, (2009) 215.

[5] M. Cordelli et al., QCALT: A tile calorimeter for KLOE-2 experiment, Nucl. Instr. and Meth. A 617 (2010) 105.

[6] F. Bossi et al. and KLOE collaboration, Precision Kaon and Hadron Physics with KLOE, Rivista del Nuovo Cimento 31 (2008).

[7] KLOE-2 collaboration, TDR of Inner Tracker for KLOE-2 experiment, LNF-10/3(P) INFN-LNF, Frascati (2010) and arXiv:1002.1557v1

[8] M. Alfonsi et al., Activity of CERN and LNF groups on large area GEM detectors Nucl. Instr. and Meth. A 604 (2009) 23.

[9] D. Babusci et al., Test of CPT and Lorentz symmetry in entangled neutral kaons with the KLOE experiment, Phys. Lett. B 730 (2014) 89.

[10] G. Amelino-Camelia et al., Physics with the KLOE-2 experiment at the upgraded $D A \Phi N E$, Eur. Phys. J C 68619 (2010) 